\title{
LPV Observer-Based Strategy for Rejection of Periodic Disturbances with Time-Varying Frequency
}

\author{
G. A. Ramos, ${ }^{1}$ Ramon Costa-Castelló, ${ }^{2}$ and John Cortés-Romero ${ }^{1}$ \\ ${ }^{1}$ Departamento de Ingeniería Eléctrica y Electrónica, Universidad Nacional de Colombia, Bogotá, Colombia \\ ${ }^{2}$ Institut d'Organització i Control de Sistemes Industrials, Universitat Politècnica de Catalunya, 08028 Barcelona, Spain
}

Correspondence should be addressed to Ramon Costa-Castelló; ramon.costa@upc.edu

Received 21 December 2014; Revised 29 April 2015; Accepted 4 May 2015

Academic Editor: Peter Dabnichki

Copyright (C) 2015 G. A. Ramos et al. This is an open access article distributed under the Creative Commons Attribution License, which permits unrestricted use, distribution, and reproduction in any medium, provided the original work is properly cited.

\begin{abstract}
Rejection of periodic disturbances is an important issue in control theory and engineering applications. Conventional strategies like repetitive control and resonant control can deal adequately with this problem but they fail when the frequency of the disturbance varies with time. This paper proposes a Linear Parameter Varying (LPV) resonant observer-based control for periodic signal rejection which is able to deal with the changes in frequency of the disturbance signal. The observer includes, in an embedded way, an internal model of the disturbance that is based on its harmonic decomposition. In this way, the frequency of the disturbance signal constitutes a parameter that can be adjusted according to the variations of the signal. The resulting disturbance estimation is then used by a control law that cancels the periodic disturbance term while controlling a specified tracking task. The proposed scheme lets the control designer address the disturbance estimation and tracking problems separately. Experimental results, on a mechatronic test bed, show that the proposed LPV resonant observer-based control successfully rejects periodic disturbances under varying frequency conditions.
\end{abstract}

\section{Introduction}

Rejection of periodic disturbances has been a subject of great interest in control theory and engineering applications. Periodic disturbances are present in many applications like robotics [1,2], power inverters [3], power active filters [4], and wind turbines [5], among others.

The most common control strategies used for rejection of periodic disturbances are repetitive control (RC) $[6,7]$ and resonant control [8]. RC constitutes a very efficient methodology in control applications that require tracking and/or rejection of periodic signals (see [9]). It is based on the internal model principle (IMP), thus requiring the inclusion of a periodic signal model in the control loop. However, one of the main drawbacks of RC appears when the frequency of the signals is uncertain or varies with time. In these cases, the traditional RC suffers from a significant performance loss [10]. In order to solve this problem, different strategies have been reported in the literature: a variable structure $\mathrm{RC}$ has been proposed in [11], where the frequency of the internal model is adapted to follow the exogenous signal changes; a High Order
Repetitive Controller (HORC) is presented in [12] which is robust against frequency variations and $[13,14]$ propose a varying sampling controller for which the frequency discrete representation remains invariant. Similarly, based on the IMP, the resonant control [15] is dedicated to the tracking/rejection of selected harmonics present in a given signal.

Alternatively, this problem can be treated using an observer-based control scheme. Under this approach, the observer is in charge of obtaining an estimate of the disturbance that is then used by the control law to reject the real disturbance. A review of disturbance observers design can be found in [16].

In this paper an LPV observer-based strategy is proposed aimed at rejecting periodic disturbances under variable frequency conditions. The proposed observer is formulated such that it includes an internal model of a periodic signal. This internal model is built from the decomposition of the periodic signal on its harmonic components. Thus, the observer is able to estimate the states of the plant and each of the selected frequency components. To overcome the frequency variation problem, the frequency of the signal, which is structurally 
embedded in the observer, is changed according to the exogenous variations. Furthermore, the observer gains are reaccommodated according to the different operating points caused by the varying frequency. The tuning of the system is a combined methodology that uses the pole placement technique for reference tracking and optimal Kalman-Bucy approach for the configuration of the resonant observer. Finally, stability analysis can be formulated in an LPV systems framework. In this way, since the closed-loop system is affine with respect to the varying frequency parameter a simple condition to establish the stability can be stated.

The experimental validation of the proposal is carried out in a mechatronic platform. This is based on a DC motor exposed to a rotating periodic disturbance torque. Experimental results show that the proposed approach exhibits very high performance, reducing effectively the effect of the disturbances over the angular speed. It is also shown that the performance is preserved under variations of the disturbance frequency. Unlike classic control schemes for handling periodic signals, resonant and repetitive control, the proposed architecture offers the advantage of independently designing the disturbance rejection and tracking reference signals.

This paper is organized as follows. Section 2 describes the architecture of the proposed controller, Section 3 describes the platform and the experimental results, and finally conclusions and future work are proposed in Section 4.

\section{Structure of Resonant Observer-Based Control}

This section describes the controller architecture of the proposed LPV observer-based strategy. The controller is composed by a disturbance observer, a state feedback control, and the reference internal model. The disturbance estimation is used to compensate the disturbance signal using the Active Disturbance Rejection Control (ADRC) philosophy. A complete stability analysis and some tuning criteria are provided.

2.1. Plant Model. Consider the following state-space linear plant model:

$$
\begin{aligned}
\dot{\mathbf{x}}_{p} & =\mathbf{A}_{p} \mathbf{x}_{p}+\mathbf{B}_{p} u+\mathbf{B}_{p} \xi_{p}, \\
y & =\mathbf{C}_{p} \mathbf{x}_{p},
\end{aligned}
$$

where $\mathbf{x}_{p} \in \mathbb{R}^{n}$ is the state vector, $u \in \mathbb{R}$ is the control action, $\xi_{p} \in \mathbb{R}$ is the disturbance signal, and $y \in \mathbb{R}$ is the system output. Similarly, $\mathbf{A}_{p} \in \mathbb{R}^{n \times n}$ is the state transition matrix, $\mathbf{B}_{p} \in \mathbb{R}^{n \times 1}$ is the input vector, and $\mathbf{C}_{p} \in \mathbb{R}^{1 \times n}$ is the output vector. The system defined by $\left(\mathbf{A}_{p}, \mathbf{B}_{p}, \mathbf{C}_{p}, \mathbf{0}\right)$ is assumed to be a minimal representation being both controllable and observable.

2.2. Disturbance Model. In this work we are dealing with disturbances that can be written as

$$
\xi=\xi_{1}+\xi_{2}+\cdots+\xi_{m}
$$

with

$$
\xi_{k}=g_{k} \sin \left(\omega_{k}(t) t+\phi_{k}\right)
$$

where $\omega_{k}(t)$ is the frequency of each component and $g_{k}$ and $\phi_{k}$ are assumed unknown. In this work it is assumed that $g_{k}$ and $\phi_{k}$ are constant or piecewise constant. Under this hypothesis the frequency content of $\xi$ is locally concentrated around $\omega_{k}(t)$.

Although the values of $\omega_{k}(t)$ might be arbitrarily assigned, a particular case with great relevance is when $\omega_{k}(t)=k$. $2 \pi / T_{p}(t)$, where $k=1, \ldots, m$. This case will be assumed from now in this work. Consequently $\omega(t)$ is defined as $\omega(t)=$ $2 \pi / T_{p}(t)$ and $\omega_{k}(t)=k \cdot \omega(t)$. In case $T_{p}(t)$ is constant, $\xi$ is a $T_{p}$-periodic signal and $\xi_{k}$ are the different harmonic frequency components.

Each sinusoidal term can be thought as generated by the following system, with appropriate initial conditions:

$$
\ddot{\xi}_{k}=-k^{2} \omega^{2}(t) \xi_{k},
$$

where $\omega$ is the fundamental frequency which is assumed measurable (or known). In state-space this can be written as

$$
\begin{aligned}
& \dot{\mathbf{z}}_{k}=\omega(t) \mathbf{A}_{k} \mathbf{z}_{k}, \\
& \xi_{k}=\mathbf{C}_{k} \mathbf{z}_{k}
\end{aligned}
$$

with $\mathbf{z}_{k} \in \mathbb{R}^{2}, \mathbf{A}_{k}=\left[\begin{array}{cc}0 & k \\ -k & 0\end{array}\right]$, and $\mathbf{C}_{k}=[1,0]$.

Therefore, the disturbance signal, $\xi$, admits the following state-space representation:

$$
\begin{aligned}
& \dot{\mathbf{z}}=\omega(t) \mathbf{A}_{z} \mathbf{z}, \\
& \xi=\mathbf{C}_{z} \mathbf{z},
\end{aligned}
$$

where $\mathbf{z}=\left[\begin{array}{llll}\mathbf{z}_{1}^{T} & \mathbf{z}_{2}^{T} & \cdots & \mathbf{z}_{m}^{T}\end{array}\right]^{T} \in \mathbb{R}^{2 m}$ and

$$
\begin{aligned}
\mathbf{A}_{z} & =\left[\begin{array}{ccccc}
\mathbf{A}_{1} & \mathbf{0} & \mathbf{0} & \cdots & \mathbf{0} \\
\mathbf{0} & \mathbf{A}_{2} & \mathbf{0} & \cdots & \mathbf{0} \\
\mathbf{0} & \mathbf{0} & \mathbf{A}_{3} & \cdots & \mathbf{0} \\
\vdots & \vdots & \vdots & \ddots & \vdots \\
\mathbf{0} & \mathbf{0} & \mathbf{0} & \cdots & \mathbf{A}_{m}
\end{array}\right], \\
\mathbf{C}_{z} & =\left[\begin{array}{lllll}
\mathbf{C}_{1} & \mathbf{C}_{2} & \mathbf{C}_{3} & \cdots & \mathbf{C}_{m}
\end{array}\right] .
\end{aligned}
$$

2.3. Augmented System. We can extend the plant model to include the disturbance signal using $\mathbf{x}=\left[\begin{array}{ll}\mathbf{x}_{p}^{T} & \mathbf{z}^{T}\end{array}\right]^{T} \in \mathbb{R}^{n+2 m}$; thus we obtain the following augmented model:

$$
\begin{aligned}
& \dot{\mathbf{x}}=\mathbf{A}(t) \mathbf{x}+\mathbf{B} u, \\
& y=\mathbf{C} \mathbf{x},
\end{aligned}
$$


where

$$
\begin{aligned}
\mathbf{A}(t) & =\left[\begin{array}{cc}
\mathbf{A}_{p} & \mathbf{B}_{p} \mathbf{C}_{z} \\
\mathbf{0} & \omega(t) \cdot \mathbf{A}_{z}
\end{array}\right], \\
\mathbf{B} & =\left[\begin{array}{c}
\mathbf{B}_{p} \\
\mathbf{0}
\end{array}\right], \\
\mathbf{C} & =\left[\begin{array}{ll}
\mathbf{C}_{\mathbf{p}} & \mathbf{0}
\end{array}\right] .
\end{aligned}
$$

This system, with appropriate initial conditions, is equivalent to (1) subject to (2) and (3). It is important to notice that (8) has no disturbance input and it is an observable system but it is not completely controllable system. The controllable subsystem corresponds to the plant while the noncontrollable subsystem corresponds to the disturbance model. Note that the disturbance is an exogenous signal and consequently it cannot be modified through the control action.

2.4. Resonant Observer. In order to observe the state of (8) a Luenberger observer is proposed:

$$
\begin{aligned}
\dot{\hat{\mathbf{x}}} & =\mathbf{A}(t) \widehat{\mathbf{x}}+\mathbf{B} u+\mathbf{L}(t)[y-\mathbf{C} \widehat{\mathbf{x}}] \\
& =(\mathbf{A}(t)-\mathbf{L}(t) \mathbf{C}) \widehat{\mathbf{x}}+\mathbf{B} u+\mathbf{L}(t) y,
\end{aligned}
$$

where $\widehat{\mathbf{x}}=\left[\begin{array}{ll}\widehat{\mathbf{x}}_{p}^{T} & \widehat{\mathbf{z}}^{T}\end{array}\right]^{T}$ is the augmented system state estimation and $\mathbf{L}(t)=\left[\begin{array}{ll}\mathbf{L}_{p}^{T}(t) & \mathbf{L}_{z}^{T}(t)\end{array}\right]^{T}$ are the observer gains.

The estimation error is defined as follows: $\mathbf{e}=\widehat{\mathbf{x}}-\mathbf{x}$. Therefore, using (8) and (10) the estimation error evolution can be written as

$$
\dot{\mathbf{e}}=[\mathbf{A}(t)-\mathbf{L}(t) \mathbf{C}] \mathbf{e} .
$$

2.4.1. Stability Analysis. The stability of system (11) depends on the matrix:

$$
\begin{aligned}
\mathbf{H}(t) & =[\mathbf{A}(t)-\mathbf{L}(t) \mathbf{C}] \\
& =\left[\begin{array}{cc}
\mathbf{A}_{p}-\mathbf{L}_{p}(t) \mathbf{C}_{p} & \mathbf{B}_{p} \mathbf{C}_{z} \\
-\mathbf{L}_{z}(t) \mathbf{C}_{p} & \omega(t) \cdot \mathbf{A}_{z}
\end{array}\right] .
\end{aligned}
$$

In order to prove the stability of system (11), a Lyapunov function can be formulated:

$$
V_{e}=\frac{1}{2} \mathbf{e}^{T} \mathbf{P}_{e}(t) \mathbf{e}, \quad \mathbf{P}_{e}(t)>\mathbf{0} .
$$

Consequently in order to guarantee closed-loop stability the following inequality must be fulfilled:

$$
\begin{aligned}
\dot{V}_{e} & =\mathbf{e}^{T} \mathbf{P}_{e}(t) \mathbf{H}(t) \mathbf{e}+\mathbf{e}^{T} \mathbf{H}(t)^{T} \mathbf{P}_{e}(t) \mathbf{e}+\mathbf{e}^{T} \dot{\mathbf{P}}_{e}(t) \mathbf{e} \\
& <\mathbf{0},
\end{aligned}
$$

so it is necessary that

$$
\mathbf{P}_{e}(t) \mathbf{H}(t)+\mathbf{H}(t)^{T} \mathbf{P}_{e}(t)+\dot{\mathbf{P}}_{e}(t)<\mathbf{0}, \quad \forall t>0 .
$$

Defining

$$
\mathbf{P}_{e}(t)=\mathbf{P}_{0}+\omega(t) \mathbf{P}_{1}
$$

where $\mathbf{P}_{0}$ and $\mathbf{P}_{1}$ are two symmetric matrices, the stability condition can be stated as

$$
\mathbf{P}_{e}(t) \mathbf{H}(t)+\mathbf{H}(t)^{T} \mathbf{P}_{e}(t)+\dot{\omega}(t) \mathbf{P}_{1}<\mathbf{0}, \quad \forall t>0 .
$$

Using LPV theory $[17,18]$, this condition can be checked in terms of an LMI which must be evaluated in four points defined by $\omega \in\left\{\omega_{\min }, \omega_{\max }\right\}$ and $\dot{\omega} \in\left\{\dot{\omega}_{\min }, \dot{\omega}_{\max }\right\}$.

2.4.2. Tuning Procedure. In Section 2.4.1 observer stability conditions have been established. These conditions do not uniquely determine the observer gain, $\mathbf{L}(t)$. An approach which provides a simple and convenient framework is optimal estimation.

Although a theory for optimal estimation for timevarying systems exists [19], it implies difficult implementation and it is not easy to apply in practice. In Linear Time Invariant framework, it is well-known that the Kalman-Bucy filter constitutes the optimal Luenberger observer where the estimation error covariance is minimized [19]. Thus, the optimal observer gain is defined by

$$
\mathbf{L}=\mathbf{P C}^{T} \mathbf{V}^{-1},
$$

where $\mathbf{P}$ is the unique positive-semidefinite solution of the algebraic Riccati equation:

$$
\mathbf{P A}^{T}+\mathbf{A P}-\mathbf{P C}^{T} \mathbf{V}^{-1} \mathbf{C P}+\mathbf{W}=\mathbf{0},
$$

where $\mathbf{V}$ and $\mathbf{W}$ are the spectral density matrices of the measurement and process noise, respectively. In this work an optimal tuning, based on the Kalman filter, is proposed. The observer gain is proposed to be linear varying as

$$
\mathbf{L}(\omega(t))=\mathbf{L}_{0}+\mathbf{L}_{1} \omega(t),
$$

where $\mathbf{L}_{0}$ and $\mathbf{L}_{1}$ are obtained by forcing $\mathbf{L}\left(\omega_{\max }\right)=\mathbf{L}_{\max }$ and $\mathbf{L}\left(\omega_{\min }\right)=\mathbf{L}_{\min }$. Thus, $\mathbf{L}_{\max }$ and $\mathbf{L}_{\text {min }}$ are obtained from (18) for $\omega_{\max }$ and $\omega_{\min }$, respectively.

Proposed approach guarantees stability at the extreme values of $\omega(t)$. Stability at intermediate points must be checked through conditions established in Section 2.4.1.

2.5. Closed-Loop System. In this section, the closed-loop stability of the system obtained using the observer estimation to close the loop is analyzed. Based on the state estimation, a state feedback control law is used; taking this into account the complete system takes the following form:

$$
\begin{aligned}
\dot{\mathbf{x}} & =\mathbf{A}(t) \mathbf{x}+\mathbf{B} u, \\
\dot{\hat{\mathbf{x}}} & =(\mathbf{A}(t)-\mathbf{L}(t) \mathbf{C}) \widehat{\mathbf{x}}+\mathbf{B} u+\mathbf{L}(t) y, \\
u & =-\mathbf{K} \widehat{\mathbf{x}},
\end{aligned}
$$

where $\mathbf{K}=\left[\mathbf{K}_{p}, \mathbf{C}_{z}\right]$ corresponds to the state feedback gain. 


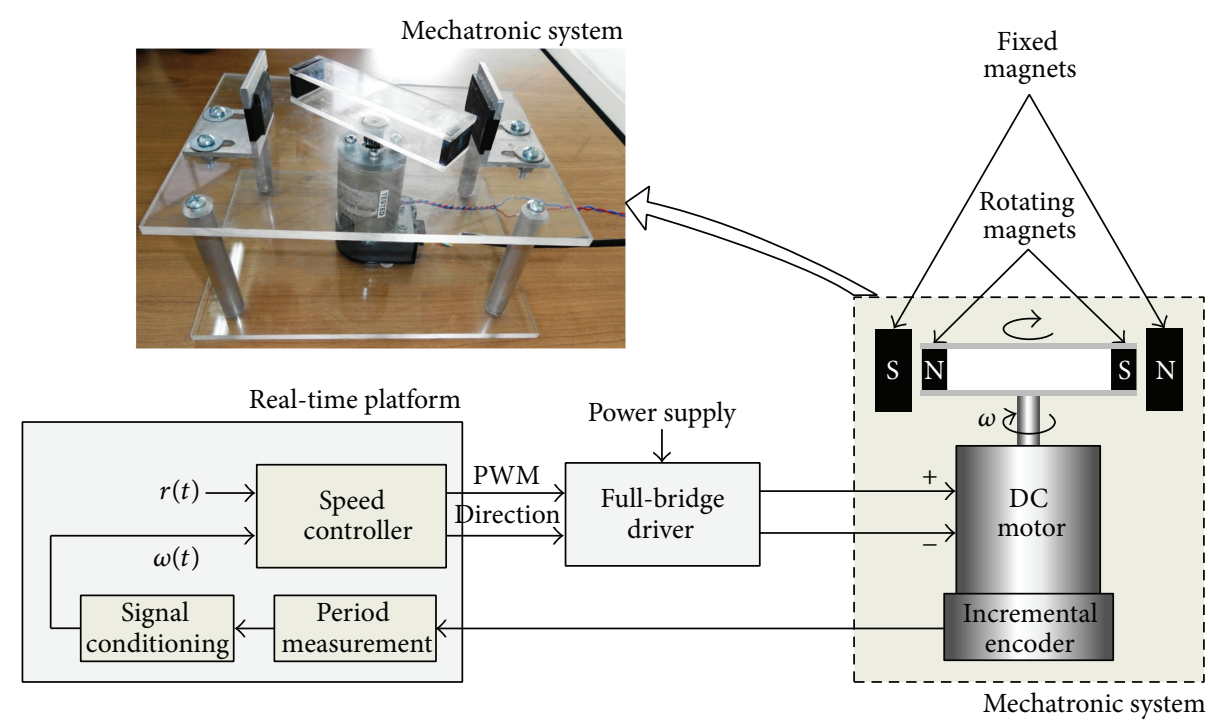

FIGURE 1: General scheme and experimental setup of the case study.

In order to simplify the analysis, these equations are written using the estimation error:

$$
\begin{aligned}
\dot{\mathbf{x}} & =\mathbf{A}(t) \mathbf{x}+\mathbf{B} u, \\
\dot{\mathbf{e}} & =(\mathbf{A}(t)-\mathbf{L}(t) \mathbf{C}) \mathbf{e}, \\
u & =-\mathbf{K}(\mathbf{x}-\mathbf{e})
\end{aligned}
$$

obtaining

$$
\begin{aligned}
\dot{\mathbf{x}} & =[\mathbf{A}(t)-\mathbf{B K}] \mathbf{x}+\mathbf{B K e}, \\
\dot{\mathbf{e}} & =(\mathbf{A}(t)-\mathbf{L}(t) \mathbf{C}) \mathbf{e} .
\end{aligned}
$$

As it can be seen the well-known separation principle can also be applied to this LPV system. The dynamics of $\mathbf{x}$ depends on

$$
[\mathbf{A}(t)-\mathbf{B K}]=\left[\begin{array}{cc}
\mathbf{A}_{p}-\mathbf{B}_{p} \mathbf{K}_{p} & \mathbf{0} \\
\mathbf{0} & \omega(t) \mathbf{A}_{z}
\end{array}\right],
$$

which can be decomposed on the periodic signal generator dynamics and the closed-loop plant dynamics. Note that the closed-loop plant dynamics is described by an LTI system dynamics and only depends on $\mathbf{A}_{p}-\mathbf{B}_{p} \mathbf{K}_{p}$, which can be stabilized with a suitable selection of the constant gain $\mathbf{K}_{p}$.

2.6. Closed-Loop System including Reference Internal Model. The controller which has been introduced in the previous section will be useful if we are interested in stabilizing the origin, but in most cases we are interested in tracking a reference. In order to guarantee this, the reference internal model will be introduced in the controller (see Figure 3):

$$
\begin{aligned}
\dot{\mathbf{x}}_{\mathrm{im}} & =\mathbf{A}_{\mathrm{im}} \mathbf{x}_{\mathrm{im}}+\mathbf{B}_{\mathrm{im}}(r-y), \\
u & =\mathbf{K}_{\mathrm{im}} \mathbf{x}_{\mathrm{im}}-\mathbf{K} \widehat{\mathbf{x}} ;
\end{aligned}
$$

with this new control law the complete closed-loop system is defined by

$$
\begin{aligned}
\dot{\mathbf{x}} & =[\mathbf{A}(t)-\mathbf{B K}] \mathbf{x}+\mathbf{B} \mathbf{K}_{\mathrm{im}} \mathbf{x}_{\mathrm{im}}+\mathbf{B K e}, \\
\dot{\mathbf{x}}_{\mathrm{im}} & =-\mathbf{B}_{\mathrm{im}} \mathbf{C} \mathbf{x}+\mathbf{A}_{\mathrm{im}} \mathbf{x}_{\mathrm{im}}+\mathbf{B}_{\mathrm{im}} r \\
\dot{\mathbf{e}} & =(\mathbf{A}(t)-\mathbf{L}(t) \mathbf{C}) \mathbf{e}
\end{aligned}
$$

The stability of this system can be analyzed in terms of the observer dynamics, $\mathbf{A}(t)-\mathbf{L}(t) \mathbf{C}$, and the matrix,

$$
\left[\begin{array}{cc}
\mathbf{A}_{p}-\mathbf{B}_{p} \mathbf{K}_{p} & \mathbf{B}_{p} \mathbf{K}_{\mathrm{im}} \\
-\mathbf{B}_{\mathrm{im}} \mathbf{C}_{p} & \mathbf{A}_{\mathrm{im}}
\end{array}\right]
$$

Note that this matrix is time invariant so it can be analyzed using regular methods, and $\mathbf{K}_{\mathrm{im}}$ and $\mathbf{K}_{p}$ can be tuned using LTI methods.

\section{Case Study}

The system used for the experimental validation of the proposed control strategy is a mechatronic system affected by nonlinear periodic disturbances. It consists of a Pulse Width Modulation (PWM) electronic amplifier, a DC motor, a 500 Pulses Per Revolution (PPR) incremental encoder, and a magnetic setup that generates a periodic torque under constant angular speed, $\omega(t)$. This disturbance torque applied to the plant is a nonlinear function of the angular position, $\theta(t)$. Hence, the control objective is to regulate the angular speed of the motor to a desired value despite the periodic torque disturbance. A detailed scheme of the mechatronic system, control loop, and the experimental setup can be observed in Figure 1. The reader is encouraged to read [20] for more detailed explanation of this system (roto-magnet plant). 


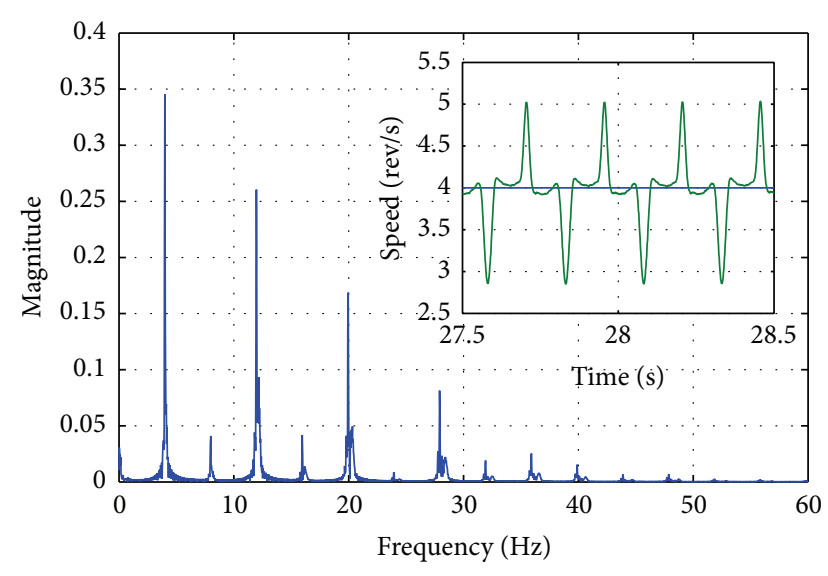

FIGURE 2: Open-loop system speed response and harmonic components at $4 \mathrm{rev} / \mathrm{s}$.

A dynamic model of the plant can be described as

$$
\begin{aligned}
\frac{d \omega(t)}{d t}= & -\frac{k_{i} k_{b}}{J R_{a}} \omega(t)-\frac{B}{J} \omega(t)+\frac{2 \pi k_{i} k_{\mathrm{pwm}}}{J R_{a}} u(t) \\
& -\frac{2 \pi}{J} \tau_{d}(\theta(t)),
\end{aligned}
$$

where $\omega(t)$ is the angular speed of the motor in rev/s, $\theta(t)$ is the angular position in $\mathrm{rad}, u(t)$ is the control input in PWM percentage with range $[-100,100], k_{\text {pwm }}=0.12 \mathrm{~V} / \%$ is the conversion constant from PWM percentage to volts, $k_{i}$ is the torque constant, $k_{b}$ is the back-emf constant, $R_{a}$ is the armature resistance, $J$ is the rotor inertia, $B$ is the viscous-friction coefficient, and $\tau_{d}(\theta(t))$ is the disturbance torque, which in this case is the nonlinear periodic torque produced by the magnetic setup (see the Appendix). Given the structural arrangement of the mechatronic system, the periodic perturbation varies according to the rotational speed of the rotating magnets. Such a time-varying disturbance is typical of rotary systems with eccentricity and imbalance problems among other typical problems.

From a simple experimental open-loop step response of the mechatronic system (free of periodic torque), the transfer function of the plant was identified as

$$
G_{p}(s)=\frac{\Omega(s)}{U(s)}=\frac{1.432}{s+1.613}
$$

then $\left(2 \pi k_{i} k_{\text {pwm }} / J R_{a}\right)=1.432$ and $\left(k_{i} k_{b} / J R_{a}+B / J\right)=1.613$.

\section{Experimental Results}

The plant system is defined by (30); thus the state-space has the form of (1) with $\mathbf{A}_{p}=-1.613, \mathbf{B}_{p}=1.432$, and $\mathbf{C}_{p}=1$.

Figure 2 shows the open-loop response of the system at $4 \mathrm{rev} / \mathrm{s}$. The speed time response and frequency spectrum are depicted. It can be seen that the disturbance torque significantly affects the speed response causing a large number of harmonic components in the speed signal.
The reference signals used in the experiments are of step and ramp types; therefore, the reference internal model is designed to have two integrators; that is, $\mathbf{A}_{\mathrm{im}}=\left[\begin{array}{ll}0 & 1 \\ 0 & 0\end{array}\right], \mathbf{B}_{\mathrm{im}}=$ $\left[\begin{array}{l}0 \\ 1\end{array}\right]$. The controller follows the structure depicted in Figure 3.

It can be noticed that the frequency of the disturbance is indirectly calculated using the reference signal. For this reason, in order to obtain a good estimation of the real frequency a suitable tracking performance is required. In other applications a different frequency estimation/calculation method may be required. As shown in Section 2.5, the observer and the feedback controller can be designed independently due to the separation principle. The feedback controller is designed to place the closed-loop poles at $-40 \mathrm{rad} / \mathrm{s}$ which is achieved

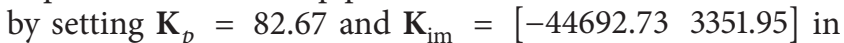
control law (26). Proposed closed-loop poles are defined in order to provide fast tracking convergence while preserving the robustness margins.

Looking at the disturbance spectrum (Figure 2) it has been determined that at least 15 resonant elements will be necessary to reject it. The selected frequencies are the fundamental frequency ( $\omega=2 \pi f \mathrm{rad} / \mathrm{s})$ and the subsequent 14 harmonic frequency components $\left(\omega_{k}=2 \pi \cdot f \cdot k \mathrm{rad} / \mathrm{s}\right.$ with $k=2, \ldots, 14)$, where $f$ is the frequency in $\mathrm{Hz}$ which corresponds with the speed value in rev/s.

The procedure to select the speed dependent observer gains is as follows:

(1) The minimum and maximum value of the speed is determined, in this case $v_{\min }=2 \mathrm{rev} / \mathrm{s}$ and $v_{\max }=$ $8 \mathrm{rev} / \mathrm{s}$, which corresponds with $\omega_{\min }=4 \pi \mathrm{rad} / \mathrm{s}$ and $\omega_{\max }=16 \pi \mathrm{rad} / \mathrm{s}$, respectively.

(2) A selection of the observer gain is performed for each of the previous operation points. The procedure to select the observer gains follows the standard Kalman-Bucy filter design as described in Section 2.4.2. We have selected $\mathbf{V}=1$ and $\mathbf{W}=$ $\gamma\left[\begin{array}{ll}0 & \mathbf{C}_{z}\end{array}\right]^{T}\left[\begin{array}{ll}0 & \mathbf{C}_{z}\end{array}\right]$ where $\gamma$ represents the compromise between system noise and observer bandwidth. In this case, the parameter $\gamma$ has been selected such that $\gamma_{\min }=2.5 \cdot 10^{-6}$ and $\gamma_{\max }=5 \cdot 10^{-7}$ provide good disturbance rejection performance for $v_{\min }$ and $v_{\max }$, respectively. It is important to note that a higher bandwidth has been designed for higher speeds since the disturbance harmonics components are then of higher frequency. Additionally, the quantification error produced by the encoder interface affects more the speeds measurement at higher velocities.

(3) The stability of the LPV observer can be checked as described in Section 2.4.1. After fixing $\omega_{\min }=$ $4 \pi \mathrm{rad} / \mathrm{s}$ and $\omega_{\max }=16 \pi \mathrm{rad} / \mathrm{s}$, a symmetric acceleration range is imposed and beginning with a very small range it is increased until no solution is found. For this system, a frequency rate variation of $\pm 10 \mathrm{rad} / \mathrm{s}^{2}$ $\left(5 / \pi \mathrm{rev} / \mathrm{s}^{2}\right)$ has been obtained.

To address the stability checking MATLAB Robust Control Toolbox [21] has been used.

Figure 4 shows the closed-loop response of the control system with the proposed LPV observer operating at $4 \mathrm{rev} / \mathrm{s}$. 


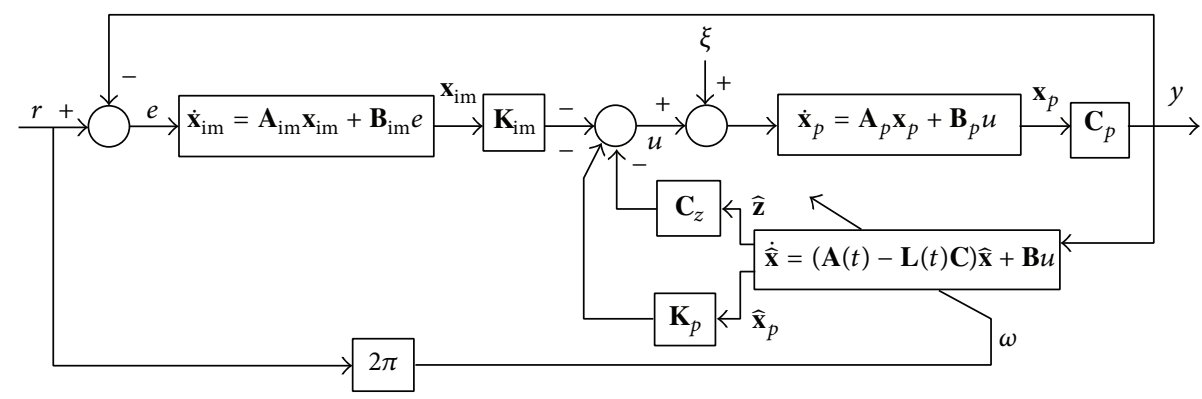

FIGURE 3: LPV observer-based control system structure.

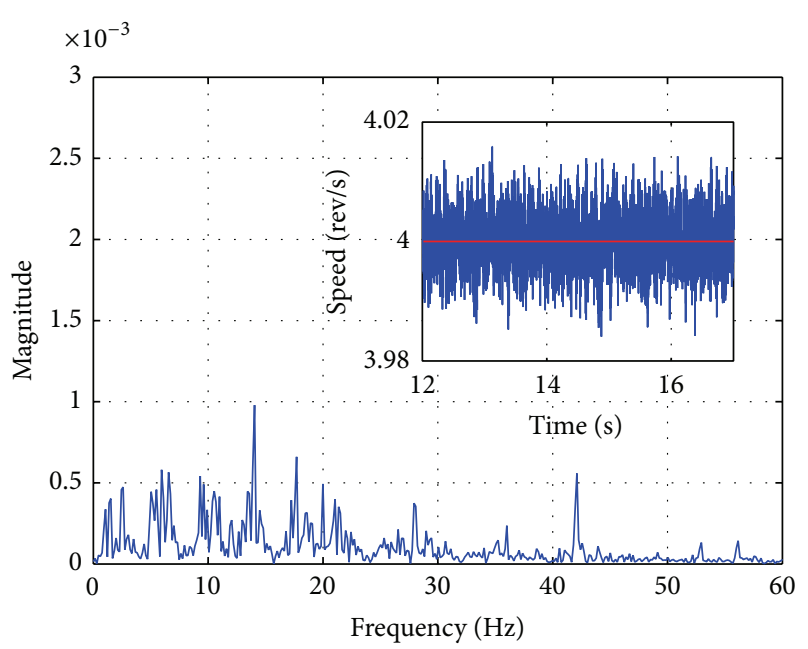

FIgURE 4: Closed-loop system speed response and harmonic components at $4 \mathrm{rev} / \mathrm{s}$.

It can be noticed that the disturbance torque has been effectively rejected, providing a constant speed with very small harmonic components. Furthermore, the obtained error lies in the noise measurement magnitude.

In the experiment, the results are obtained using a reference signal with speed variations described by

$$
r(t)= \begin{cases}4 \mathrm{rev} / \mathrm{s} & 0 \mathrm{~s} \leq t \leq 25 \mathrm{~s} \\ -\frac{1}{9} t+\frac{61}{9} \mathrm{rev} / \mathrm{s} & 25 \mathrm{~s} \leq t \leq 34 \mathrm{~s} \\ 3 \mathrm{rev} / \mathrm{s} & 34 \mathrm{~s} \leq t \leq 38 \mathrm{~s} \\ \frac{3}{17} t-\frac{63}{17} \mathrm{rev} / \mathrm{s} & 38 \mathrm{~s} \leq t \leq 55 \mathrm{~s} \\ 6 \mathrm{rev} / \mathrm{s} & t \geq 55 \mathrm{~s} .\end{cases}
$$

Two different configurations of the LPV observer have been used: a setup in which the frequency of the observer remains constant corresponding with $4 \mathrm{rev} / \mathrm{s}$ and the fully LPV observer configuration. Figure 5 presents the obtained experimental speed response. It can be seen that when the observer does not change the frequency the control system loses its performance as the speed deviates from the nominal setup. On the other side, the proposed LPV observer can
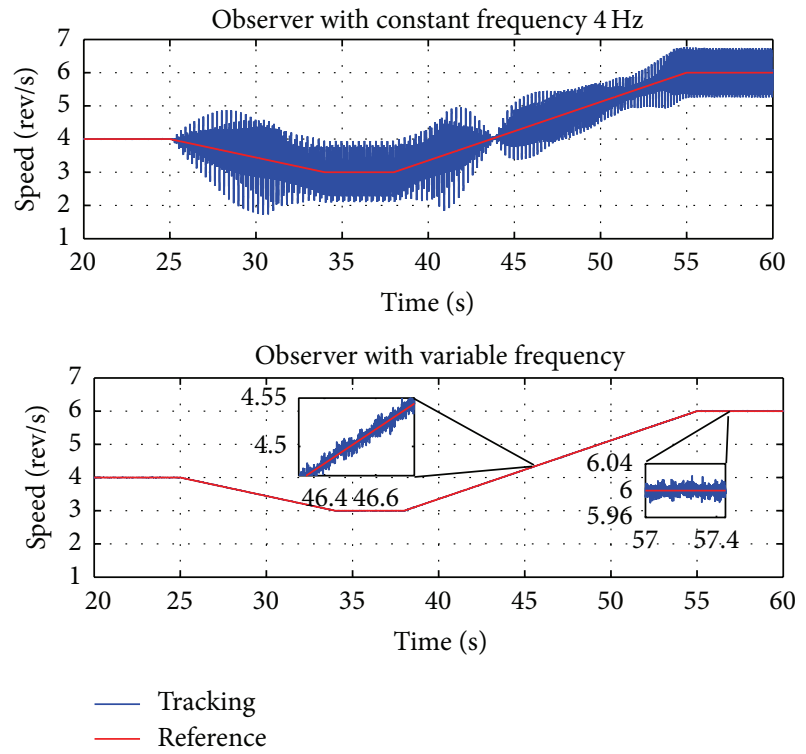

FIGURE 5: Closed-loop system speed response with variable speed.

successfully reject the disturbance for all speed variations, thus tracking the reference signal with small error. As shown in Figure 5, even during a ramp type speed variation the system obtains very small error, provided the ramp slope is small enough.

The control signal of the proposed LPV observer-based strategy is presented in Figure 6. It can be noticed that the control signal profile changes its frequency following the speed changes, thus feeding the plant with the appropriate input to compensate the time-varying disturbances. Additionally, compared with the estimated disturbance shown in Figure 7, it is noticeable that the control signal shape corresponds with the disturbance one in order to provide the obtained rejection.

\section{Concluding Remarks}

In this paper a new control architecture based on a resonant observer for estimation and rejection of periodic disturbances is proposed. The proposed observer estimates the harmonic decomposition of the disturbance and the 


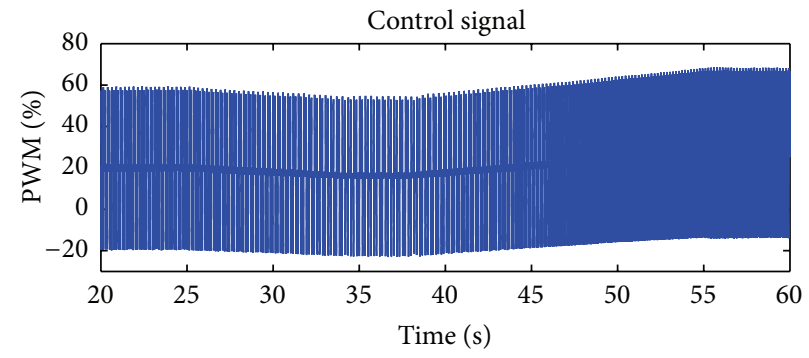

(a)
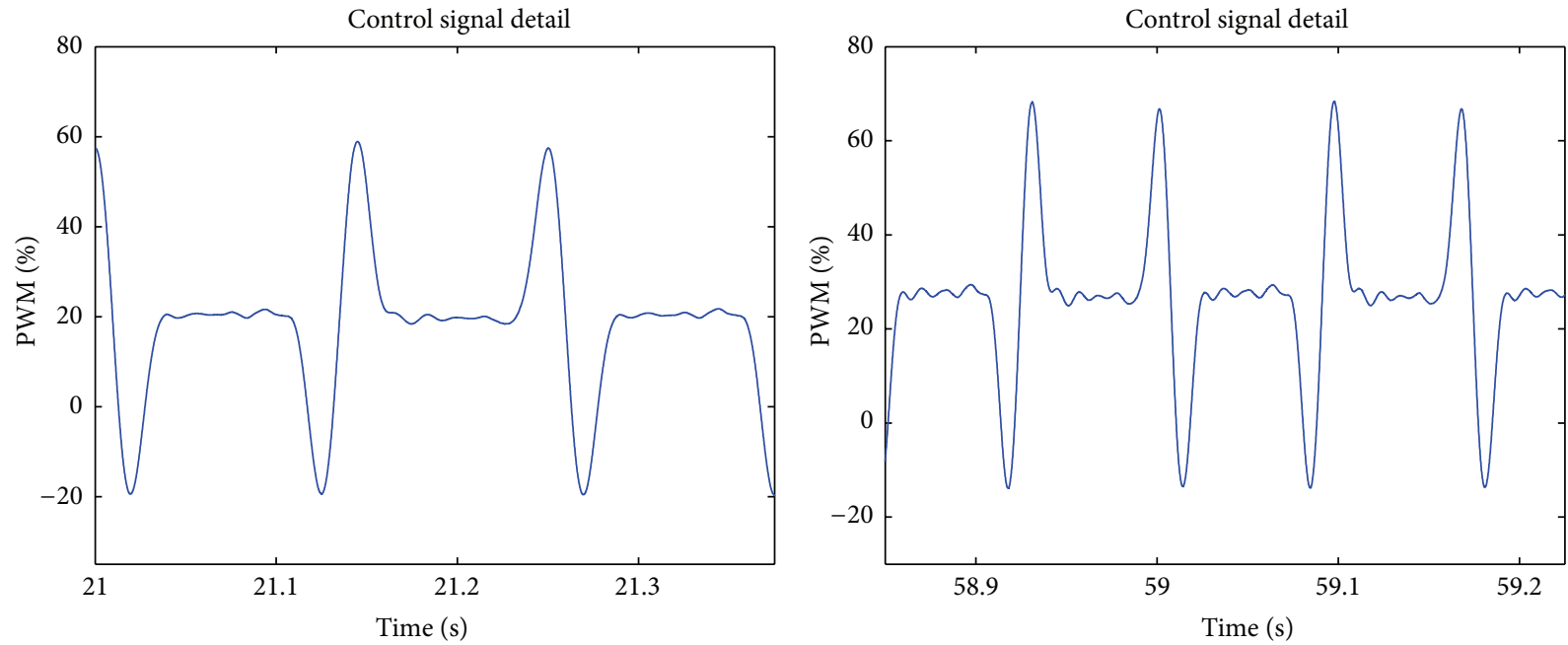

(b)

FIGURE 6: Obtained control signal using a variable speed profile. Top: the entire time interval. Bottom: detailed view for two different operating points.

controller uses this estimation to cancel its effect on the system. The controller is designed to meet further tracking tasks. LPV resonant observer tuning is performed by applying the Kalman-Bucy filter. As a result, the performance of the estimation can be adjusted by a single parameter. Experimental results show that the proposed strategy effectively rejects the periodic disturbance imposing adequate tracking error dynamics also under varying frequency conditions.

The authors are currently working to extend proposed controller to different types of electrical machines and developing new methodologies which guarantee stability in a constructive manner.

\section{Appendix}

\section{Magnetic Torque}

Since the magnetic setup of the mechatronic system is composed by two rotating magnets and two fixed magnets, the disturbance torque obeys the following nonlinear function [22]:

$$
\tau_{d}(t, \theta)=\sum_{i=1}^{2} \sum_{j=1}^{2} q_{m_{i}} m_{j} \tau_{p}\left(\theta+\theta_{i, j}, r_{i}, x_{j}\right)
$$

with

$$
\begin{aligned}
& \tau_{p}(\theta, r, x)=\frac{e}{m}\left(\sin (\rho) B_{d}+\cos (\rho) B_{\alpha}\right), \\
& \rho=\arctan (r \sin (\theta), r \cos (\theta)-x)-\theta, \\
& B_{d}=\frac{\mu_{0} m \cos (\arctan (r \sin (\theta), r \cos (\theta)-x))}{2 \pi\left(-2 r \cos (\theta) x+x^{2}+r^{2}\right)^{3 / 2}}, \\
& B_{\alpha}=\frac{\mu_{0} m \sin (\arctan (r \sin (\theta), r \cos (\theta)-x))}{4 \pi\left(-2 r \cos (\theta) x+x^{2}+r^{2}\right)^{3 / 2}},
\end{aligned}
$$

where $\theta(t)$ is the angular position of the motor in $\mathrm{rad}, q_{m_{i}}$ is the magnetic intensity of the pole for the $i$ th moving magnet, $m_{j}$ is the magnetic torque for the $j$ th fixed magnet, $r_{i}$ is the distance between the $i$ th moving magnet and its rotation axis, $x_{j}$ is the distance between the $j$ th fixed magnet and its rotation axis, $\theta_{i, j}$ is the relative position of the $i$ th moving magnet with respect to the $j$ th fixed magnet, $e$ is the thickness of each moving magnet, and $\mu_{0}$ is the magnetic constant. For simulation purposes we use $\mu_{0}=4 \pi \cdot 10^{-7} \mathrm{~N} \cdot \mathrm{A}^{-2}, e=0.004 \mathrm{~m}$, $r_{1}=0.05 \mathrm{~m}, r_{2}=0.05 \mathrm{~m}, x_{1}=0.055 \mathrm{~m}, x_{2}=0.055 \mathrm{~m}$, $\theta_{11}=0 \mathrm{rad}, \theta_{12}=\pi \mathrm{rad}, \theta_{21}=\pi \mathrm{rad}$, and $\theta_{22}=0 \mathrm{rad}$. 


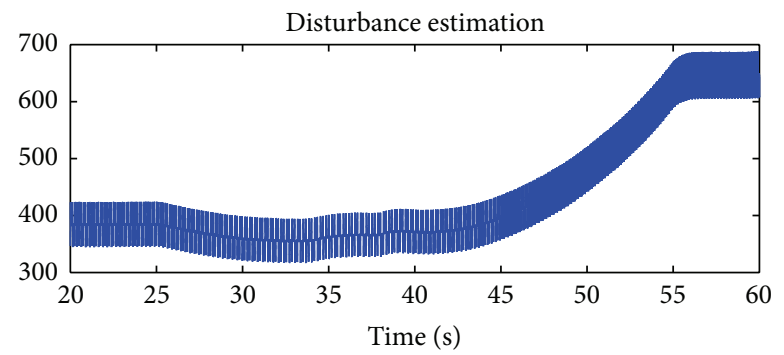

(a)
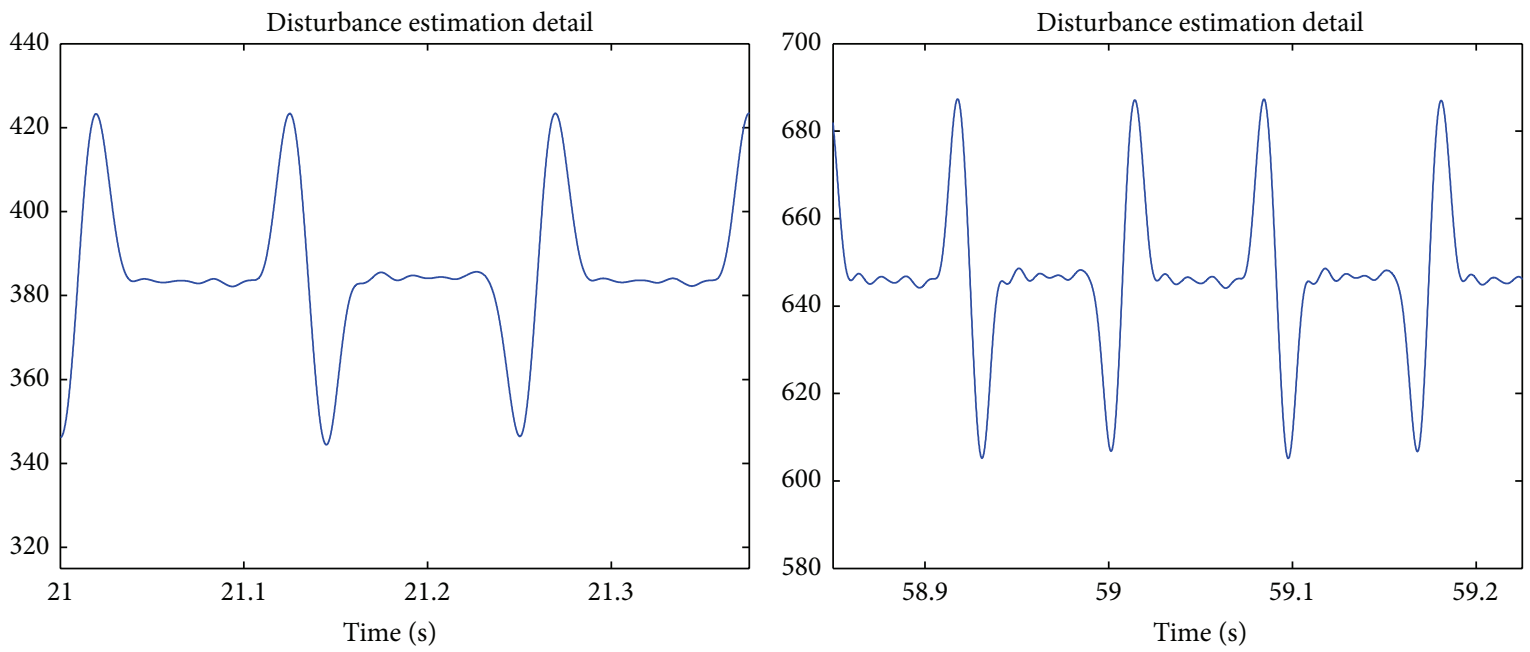

(b)

Figure 7: Disturbance estimation. Top: the estimation for the whole time interval. Bottom: detailed view of the estimation for two different operating points.

\section{Conflict of Interests}

The authors declare that there is no conflict of interests regarding the publication of this paper.

\section{Acknowledgments}

This work has been partially funded by the following project: MICINN DPI2011-25649 (financed by the Spanish Ministry of Science and Innovation and EU-ERDF funds). This work is partially supported by the Generalitat de Catalunya through Project 2014 SGR 267.

\section{References}

[1] S.-C. Wu and M. Tomizuka, "An iterative learning control design for self-servowriting in hard disk drives," Mechatronics, vol. 20, no. 1, pp. 53-58, 2010.

[2] A. Tayebi, S. Abdul, M. B. Zaremba, and Y. Ye, "Robust iterative learning control design: application to a robot manipulator," IEEE/ASME Transactions on Mechatronics, vol. 13, no. 5, pp. 608-613, 2008.

[3] B. Zhang, K. Zhou, Y. Wang, and D. Wang, "Performance improvement of repetitive controlled PWM inverters: a phaselead compensation solution," International Journal of Circuit Theory and Applications, vol. 38, no. 5, pp. 453-469, 2010.
[4] R. Griñó, R. Cardoner, R. Costa-Castelló, and E. Fossas, "Digital repetitive control of a three-phase four-wire shunt active filter," IEEE Transactions on Industrial Electronics, vol. 54, no. 3, pp. 1495-1503, 2007.

[5] I. Houtzager, J.-W. van Wingerden, and M. Verhaegen, "Rejection of periodic wind disturbances on a smart rotor test section using lifted repetitive control," IEEE Transactions on Control Systems Technology, vol. 21, no. 2, pp. 347-359, 2013.

[6] M. Tomizuka, "Dealing with periodic disturbances in controls of mechanical systems," Annual Reviews in Control, vol. 32, no. 2, pp. 193-199, 2008.

[7] R. W. Longman, "Iterative learning control and repetitive control for engineering practice," International Journal of Control, vol. 73, no. 10, pp. 930-954, 2000.

[8] Z. Shuai, A. Luo, C. Tu, and D. Liu, "New control method of injection-type hybrid active power filter," IET Power Electronics, vol. 4, no. 9, pp. 1051-1057, 2011.

[9] L. Cuiyan, Z. Dongchun, and Z. Xianyi, "A survey of repetitive control," in Proceedings of the IEEE/RSJ International Conference on Intelligent Robots and Systems (IROS '04), pp. 1160-1166, October 2004.

[10] M. Steinbuch, "Repetitive control for systems with uncertain periodtime," Automatica, vol. 38, no. 12, pp. 2103-2109, 2002.

[11] J. S. Hu, "Variable structure digital repetitive controller," in Proceedings of the American Control Conference, pp. 2686-2690, June 1992. 
[12] M. Steinbuch, S. Weiland, and T. Singh, "Design of noise and period-time robust high-order repetitive control, with application to optical storage," Automatica, vol. 43, no. 12, pp. 2086-2095, 2007.

[13] J. M. Olm, G. A. Ramos, and R. Costa-Castelló, "Adaptive compensation strategy for the tracking/rejection of signals with time-varying frequency in digital repetitive control systems," Journal of Process Control, vol. 20, no. 4, pp. 551-558, 2010.

[14] G. A. Ramos, J. M. Olm, and R. Costa-Castelló, "Digital repetitive control under nonuniform sampling: an LMI stability analysis," Mathematical Problems in Engineering, vol. 2011, Article ID 120570, 16 pages, 2011.

[15] M. F. Byl, S. J. Ludwick, and D. L. Trumper, "A loop shaping perspective for tuning controllers with adaptive feedforward cancellation," Precision Engineering, vol. 29, no. 1, pp. 27-40, 2005.

[16] A. Radke and Z. Gao, "A survey of state and disturbance observers for practitioners," in Proceedings of the American Control Conference, pp. 5183-5188, June 2006.

[17] P. Gahinet, P. Apkarian, and M. Chilali, "Affine parameterdependent Lyapunov functions and real parametric uncertainty," IEEE Transactions on Automatic Control, vol. 41, no. 3, pp. 436-442, 1996.

[18] G. Duan and H.-H. Yu, LMIs in Control Systems: Analysis, Design and Applications, Linear Matrix Inequalities in Control Systems, CRC Press, Boca Raton, Fla, USA, 2013.

[19] B. D. O. Anderson and J. B. Moore, Optimal Control: Linear Quadratic Methods, Prentice-Hall, Upper Saddle River, NJ, USA, 1990.

[20] G. A. Ramos, R. Costa-Castelló, and J. M. Olm, "Roto-magnet," in Digital Repetitive Control under Varying Frequency Conditions, vol. 446 of Lecture Notes in Control and Information Sciences, pp. 67-100, Springer, Berlin, Germany, 2013.

[21] R. Y. Chiang and M. G. Safonov, "Robust control toolbox for use with MATLAB: user's guide," Reimpression Mars 1996-SOP97-08 SOP-98-008, 1997.

[22] E. Xargay and R. Costa-Castelló, "Modelado de una planta disenada para ilustrar el principio del modelo interno," in $X X V$ Jornadas de Automatica, J. A. Somolinos, Ed., p. 9, Ciudad Real, Spain, September 2004. 


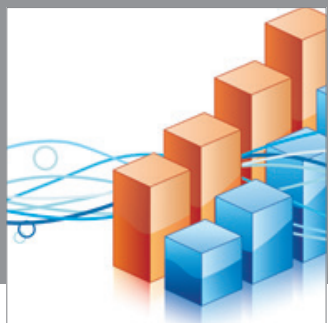

Advances in

Operations Research

mansans

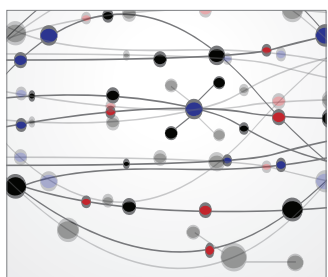

The Scientific World Journal
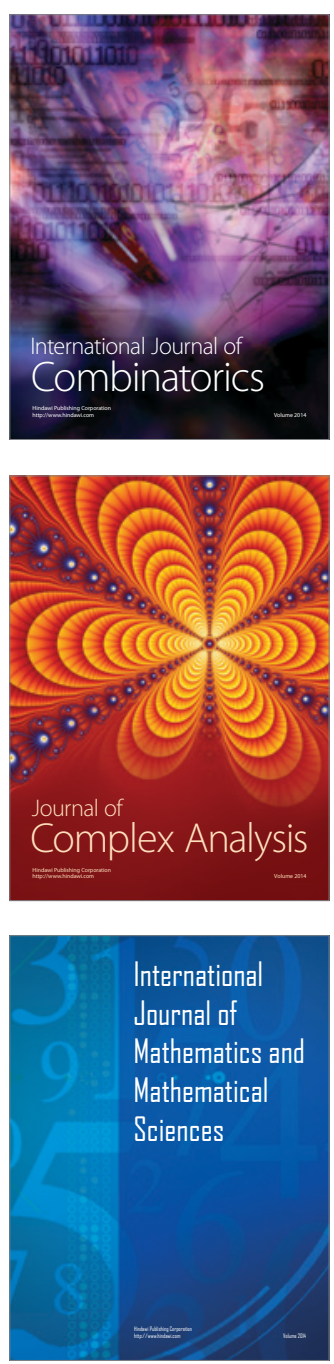
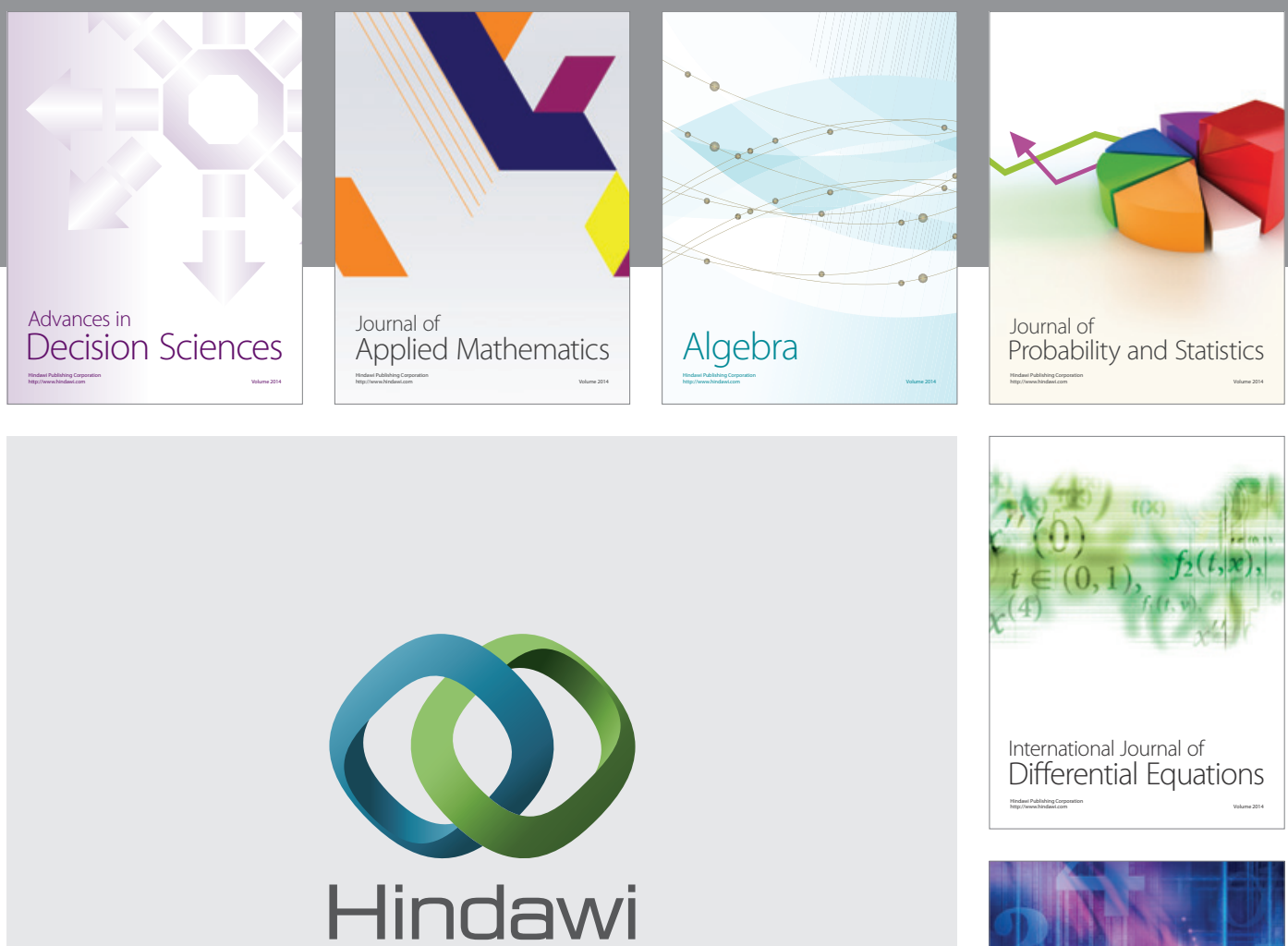

Submit your manuscripts at http://www.hindawi.com
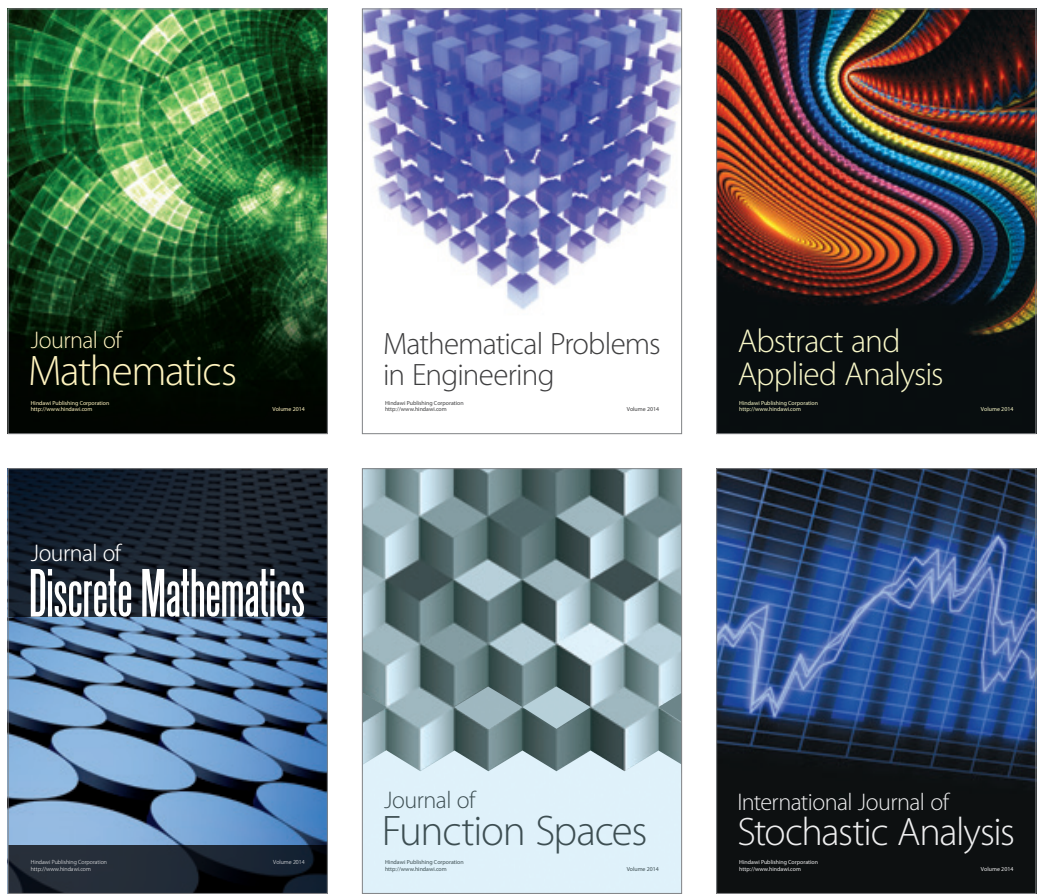

Journal of

Function Spaces

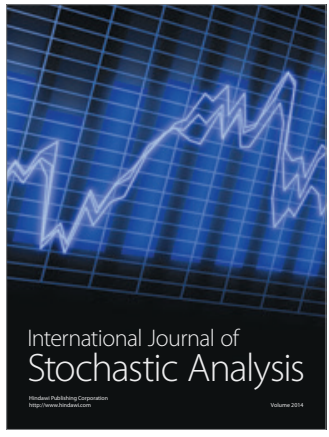

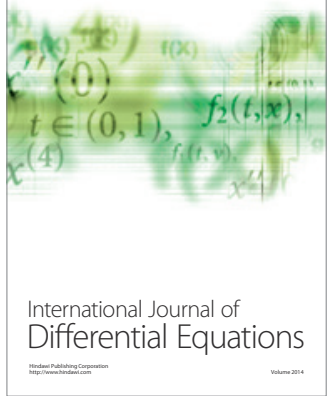
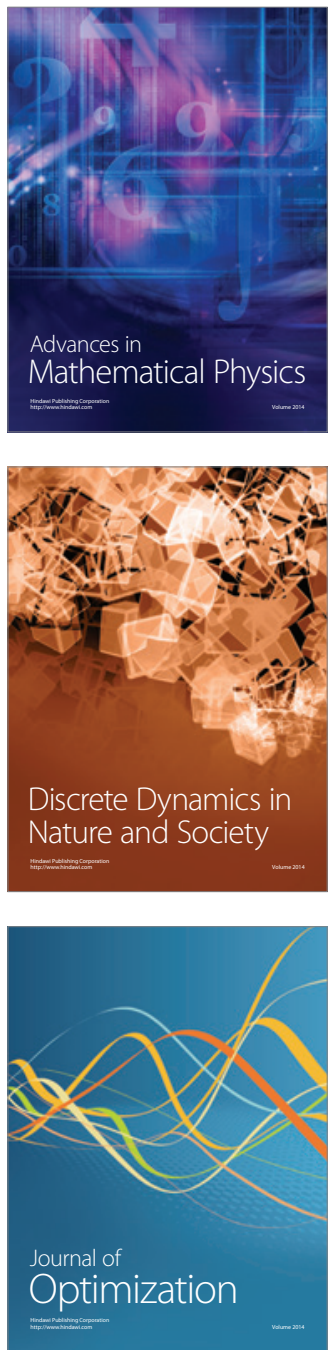